\title{
Colorectal Cancer in the Arab World - Screening Practices and Future Prospects
}

\author{
Mostafa A Arafa*, Karim Farhat
}

\begin{abstract}
Colorectal cancer (CRC) incidence and mortality rates have dropped $30 \%$ in the US in the last 10 years among adults ages 50 and older due to the widespread uptake of colonoscopy, yet incidences in the Arab countries have been increasing in the past ten years, albeit with lower figures when compared with developed countries. Lifestyle changes, food consumption patterns and obesity have been observed during the past years where the regular consumption of traditional foods is being replaced with more Western-style and ready-made foods. Most high income countries have implemented population based colorectal cancer screening programs, which aid in decreasing the incidence and mortality of cancer, while these are lacking in most of the Arab world countries due to many cultural and religious barriers to CRC screening as well as lack of high education or familiarity. What is needed is health education to modify risky lifestyle, and to increase motives and enhance positive attitudes towards early screening especially amongst high risk groups in addition to policy designed to encourage healthier living.
\end{abstract}

Keywords: Colorectal cancer - Arab world - screening - health education - incidence

Asian Pac J Cancer Prev, 16 (17), 7425-7430

\section{Introduction}

Colorectal cancer (CRC) is the third most common cancer worldwide after lung and breast cancers with two-thirds of all colorectal cancers occurring in the more developed regions of the world, with nearly 1.4 million new cases diagnosed in 2012 (World cancer research Fund International (www.wcrf.org); Dragovich and Tsikitis, 2012).There are notable differences in colorectal cancer incidences in more developed versus less developed countries.

In March 2014,American Cancer Society released data showing colon cancer incidence rates have dropped $30 \%$ in the U.S. in the last 10 years among adults ages 50 and older due to the widespread uptake of colonoscopy, with the largest decrease occurring in those ages 65 and older. In January of 2013, the American Cancer Society reported a $30 \%$ decrease in the mortality rate for colorectal cancer. There has been a decline in lives lost to cancer (1991 to 2009) and we have seen a $30 \%$ decrease in the mortality rate for colorectal cancer (Colorectal cancer, Facts \& Figures, 2014-2016 (www.cancer.org)).

\section{Figures in the Arab world}

The situation is different in the Arab world, although the figures reported for colorectal cancer is much lower than that for developed countries, yet the incidence of colorectal cancer in the Arab countries in increasing in the past ten years. Palestinian Arabs have a much lower incidence of colorectal cancer compared to Jews. One explanation for this observation has been the influence of increased amounts of fruits and vegetables in the Arabic diet. Both males and females show almost the same incidence rate. In spite of the low overall incidence of colon cancer in these countries, however, in several Gulf countries, such as Oman, Qatar, and Saudi Arabia, the incidence of colorectal cancer in people under 40 years of age has been found to be relatively high. A similar trend is also seen in Sudan, with an interesting addition of increased frequency of colorectal cancer being observed in Northern than Southern Sudan (Colon Cancer (www. cags.org.ae)).

In The Gulf countries; colorectal cancer is the second most common cancer in the Gulf cooperation countries (GCC) States. During the 10-year period, 7,641 colorectal cancer cases (8.0\% from all cancers) were reported from all GCC States. The overall ASRs for all GCC States were 7.6 and 6.5 per 100,000 populations for males and females respectively. Kuwait reported the highest incidence of colorectal cancer (15.5) among males followed by Qatar and Bahrain. Oman reported the lowest ASR in males (5.5). Qatari women had the highest incidence of colorectal cancer (ASR per 100,000 population) (16.6) followed by Kuwait and Bahrain whereas Oman reported the lowest incidence (4.1).Colorectal cancer incidence continues to increase during the ten-year period in both genders. Between 1998 and 2007, the total number of newly diagnosed colorectal cancer increased by 2.3 folds in males and 2.7 folds in females. There was observed 
increased in the trend of the age standardized incidence rate, it was significantly increased for both genders (GCC Cancer Registry, 1998-2007).

The ASR of CRC in Algeriein increasing from $10.6(1985)$ to 18 (2008) for males and from 8.5 to 13.4 for females. In Egypt, low rates of CRC (6.9/105 for males and 5.1/105 for females) were reported by the Middle East Cancer Consortium for the period of 1999-2001(Barchana, 2006). The low rates of CRC in Egypt were also reported from the Gharbiah cancer registry for the period of 2000-2002, where the age-standardized incidence rates for CRC were 6.5/105 for males and 4.2/105 for females (Veruttipong et al., 2012). According to the national cancer registry in Jordan; colorectal cancer ranked the first among males (14.7/100.00) and second among females (9.4/100.000) during 2010, the trend of ASIR if increasing through the period 1996-2009. In Lebanon Colon incidence rates ranked fourth among males and remained stable at 15.3 per 100,000, while Among females, it was the second most-common cancer at 14.1 cases per 100,000 despite a slight decrease over the six-year period (Shamseddine et al., 2014). The figures reported from Tunisia showed a significant increasing trend of colorectal cancer between 1993 and 2007 in Central Tunisia (Missaoui et al., 2011).

\section{Why colorectal cancer is increasing in the Arab world and gulf area}

Drastic changes in the lifestyle and food consumption patterns have been observed during the past years, the regular consumption of traditional foods is being replaced with more Western-style and ready-made foods. In addition to adopting a sedentary life style in daily life and other related activities. According to National Health Scurvies' in the GCC countries, high daily caloric intake, refined carbohydrates and protein were associated with increased risk of non-communicable diseases including colorectal cancer .It has been quite notable that incidence rates of cancer have increased in other countries that went through rapid industrialization because of the lifestyle risk factors including diet, physical activity, obesity which appears to play an important role in the etiology of the disease (Giovannucci, 2002).

The international experts from the World Cancer Research Fund Report, 2008, concluded that colorectal cancer is a major public health problem and modernization in food choices, sedentary life style and increased life expectancy are the major factors that might synergize with genetics for the epidemic of CRC (World Cancer Research Fund (WCRF) and American Institute for Cancer Research (AICR), 2008).

Few dietary population based studies were conducted at the Middle East, yet reported high consumption of red meat, fast foods and saturated dietary fats and a decreased consumption of fresh vegetables and fruits, a typical high risk dietary profile for CRC (Musaiger and Miladi, 2001; Galal, 2003) The study conducted by Arafa et al in Jordan indicated that CRC cases enrolled in study, were adopting a sedentary lifestyle and their dietary pattern was high frequency of red meat and saturated fat consumption and low consumption of fruits and vegetables. In addition, they had inadequate daily intake of four key nutrients that protect against CRC risk, vitamin C, selenium folate and B12 (Arafa et al., 2011).

The complexity of the human diet represents a daunting challenge to researchers contemplating a study of its relation to CRC, and very few national studies (Arafa et al., 2011, Waly et al., 2012) have tackled the role of Westernization of diet in association with CRC. Meeting energy and nutrients needs, and preventing nutritional-

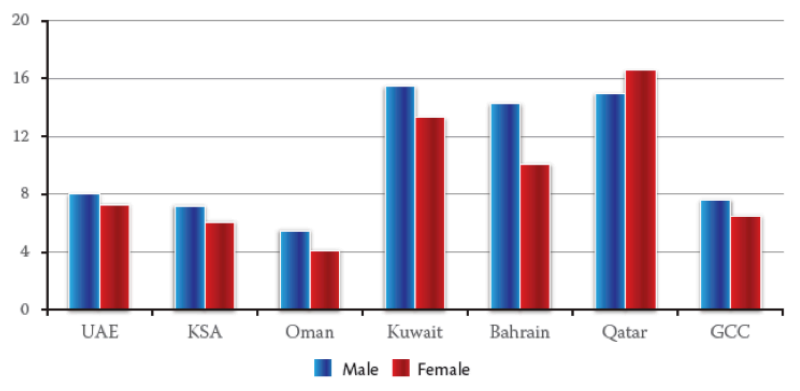

Figure 1. Age Standardized Incidence Rates (ASRs) of Colorectal Cancer in the GCC States (1998-2007)

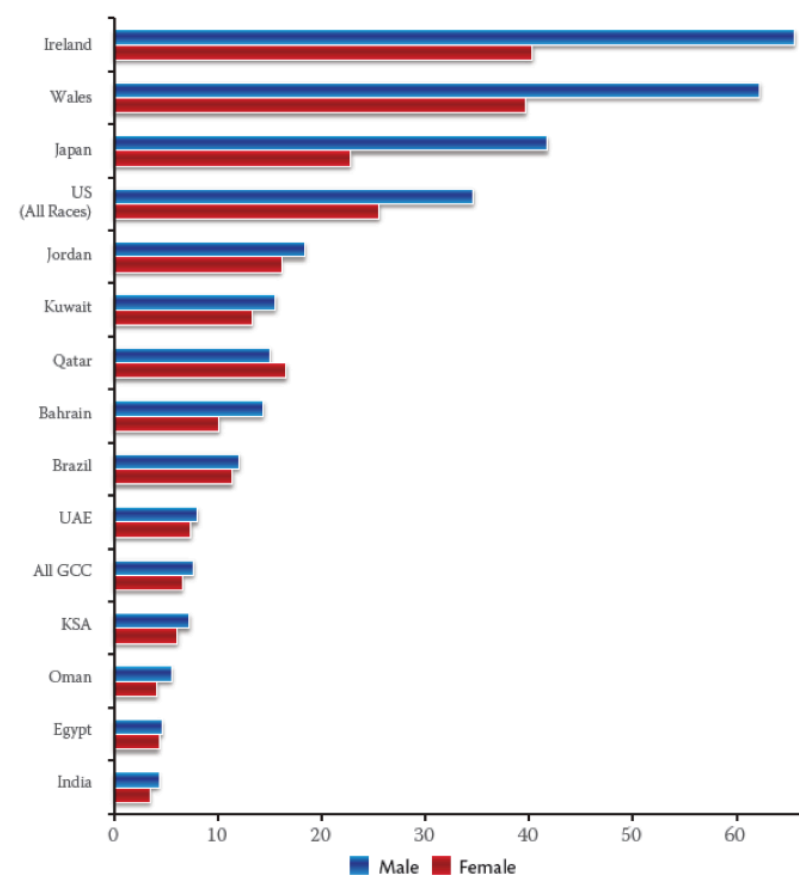

Figure 2. Comparison of ASRs of Colorectal Cancer in the GCC States with Selected Countries

Colon Cancer Cases Arising in Various Family Risk Settings

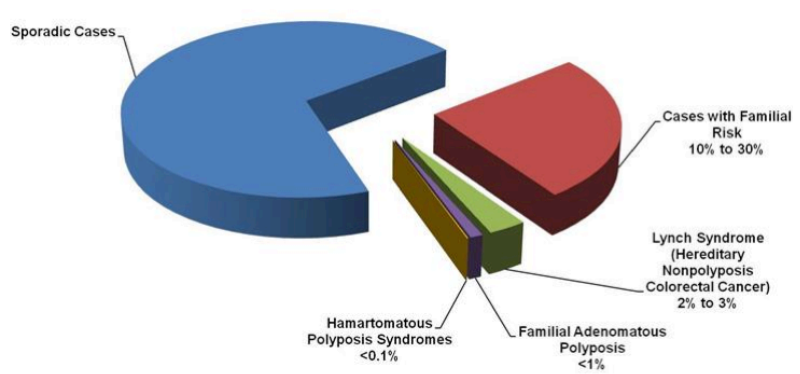

Figure 3. Fractions of Colon Cancer Cases That Arise In Various Family Risk Settings. Reprinted from Gastroenterology, Vol. 119, No. 3, Randall W. Burt, Colon Cancer Screening, Pages 837-853. 
related disorders while establishing healthy eating habits among adolescents is the main challenge for primary prevention of CRC.

In the same context, Fahad and his colleagues (Alamri et al., 2014) reported a trend of low intake of dietary antioxidants among CRC cases as compared to controls in their study in Saudi Arabia, where adequate intake of dietary antioxidants continues to play an important in the primary prevention of oxidative stress-mediated chronic diseases, including CRC (Leenders et al., 2014).

\section{Screening of Colorectal Cancer}

Screening has the potential to prevent colorectal cancer because it can detect precancerous growths, called polyps, in the colon and rectum. Although most polyps will not become cancerous, removing them can prevent cancer from occurring. Furthermore, regular screening increases the likelihood that colorectal cancers that do develop will be detected at an early stage, when they are more likely to be cured, treatment is less extensive, and recovery is faster. The strategy for screening for CRC should be assessed with respect to its effectiveness, sensitivity, the number of false positive results, safety, and comfort. Furthermore, the cost and economic factors pertaining to the screening programs should be observed in order to help patients with decision making, and the prevailing clinical policies should be taken into consideration (Dolatkhah et al., 2015; Hogan et al., 2015; Weyl et al., 2015).

Early detection is part of a cancer control strategy that includes creating awareness among the general public and more specific among first degree relatives of colorectal cancer patients through health education, improving awareness and clinical skills of health care providers, empowering people at risk and symptoms to seek prompt medical attention, availability of easily accessible, affordable and efficient health services with adequate infrastructure, human resources and information system, Prompt diagnosis and follow up care (WHO, 2002). Whereas most high income countries have implemented population based colorectal cancer screening programs, these are lacking in most of the Arab world countries. Improving awareness on cancer signs and symptoms and early diagnosis among high risk population in the most widely used and accepted strategy in many countries.

The only study that attempted to screen first degree relatives of CRC in Jordan was conducted in Jordan (Arafa et al., 2011), it yielded a net result of $2.1 \%$, positive cases. It raised also the question of cost effectiveness and cost benefits of running a nationwide screening program for colo-rectal cancer in a developing country, on the other hand it highlighted the importance of cancer early detection activities in Jordan as it was the first study to be conducted among community dwelling high risk population and how much the knowledge deficiency may significantly affect the screening behavior of high risk population.

There are many cultural and religious barriers to CRC screening; lack of high education or familiarity with CRC screening, distrust of Western medicine, religious objection, in addition to lack of patient physicians' awareness and cultural barriers specially among females in the Moslems culture towards colonoscopy . Improving our understanding of these could increase screening among Arab populations in the Middle East (Ahmed, 2013 Qumseya et al., 2014).

\section{Genetics and Colorectal Cancer Screening}

Advances in genomics is beginning to make significant impact on the diagnosis and management of sporadic and inherited colorectal adenocarcinoma (CRC) such as familial adenomatous polyposis.

$\mathrm{CRC}$ is caused by mutations in several different genes. Mutations in a single gene result in a marked predisposition to CRC in two distinct syndromes: Familial adenomatous polyposis (FAP) and hereditary nonpolyposis colorectal cancer (HNPCC) (Figure 3). Individuals with FAP have a $100 \%$ chance of developing CRC which is caused by mutations in the APC gene (Nishisho et al., 1991), whereas HNPCC is caused by mutations in several genes, including MSH2, MLH1, PMS1, PMS2, MSH6, TFGBR2, and MLH3 (Fishel et al., 1993; Papadopoulos et al., 1994; Horii et al., 1994; Nicolaides et al., 1995; Drummondet al., 1995; Markowitz et al.,1995; Lipkin et al., 2001).

There are other colorectal cancer syndromes include autosomal recessive adenomatous polyposis which is caused by mutations in the MUTYH gene (Farrington et al., 2005) and oligodontia-colorectal cancer syndrome which is caused by mutations in the AXIN2 gene (Liu et al., 2000). The CHEK2 gene has been implicated in susceptibility to CRC (Kilpivaara et al., 2006). In addition, somatic mutations in many different genes, including KRAS, PIK3CA, BRAF, CTNNB1, AXIN2, AKT1, and MCC, have been identified in colorectal cancer (Kinzler et al., 1991; Ilyas et al., 1997; Liu et al., 2000; Rajagopalan et al., 2002; Samuels et al., 2004; Carpten et al., 2007; Misale et al., 2012).

Genome-wide association studies have shown that common alleles of SMAD7 influence colorectal cancer risk (Broderick et al., 2007). Germline susceptibility loci for colorectal cancer have been mapped to chromosomes 9q22.2-q31.2 (CRCS1) (Guda et al., 2009) and 8q24 (CRCS2) (Tomlinson et al., 2007).

A recent meta-analysis using data collected from more than 12,000 participants in 6 studies performed from 1990 through 2011 in the United States (US) and Germany presents a model to determine colorectal cancer risk using common genetic susceptibility loci. The authors suggest that this model might be used to develop screening and prevention strategies (Vatandoost et al., 2015).

On the other hand, the state of DNA methylation plays a role in genetic instability in CRC. Hypomethylation can lead to oncogene activation, whereas hypermethylation can lead to silencing of tumor suppressor genes. In 2012, Drs AJAY GOEL and C. RICHARD BOLAND postulated that the tests based on gene mutations did not work because CRCs are heterogeneous genetically, and not all cancers carried the mutations that were used in the screening panel. Instead, they opted to look at DNA methylation; it is much more common, with a measurable frequency even in healthy individuals. They 
selected tumor suppressor genes (e.g., those that regulate and restrain cell growth) that are frequent targets for methylation in CRC and demonstrated that a screening test based on these markers could detect abnormal cell growth in the colon with a high degree of sensitivity. The test could distinguish polyps from carcinomas, and small polyps from larger polyps, based on the degree of methylation (Goel and Boland, 2012).

\section{Indications for Genetic Testing}

General indications for genetic testing have been proposed by the American Society of Clinical Oncology. The first indication for genetic testing is identification of an individual who has a personal or family history of cancer with features that suggest a genetic predisposition. The second indication is the existence of genetic tests whose results can be adequately interpreted. The third indication is that the test results will influence the management of the patient or at-risk family members (American Society of Clinical Oncology, 2003).

In conclusion, whether genetic tests will replace or supplement confirmed methods of screening has yet to be confirmed. Suitable procedures and use of genetic testing requires clinical recognition of the familial cancer syndromes and professional genetic counseling.

\section{How to Increase Colorectal Cancer Screening Uptake in the Arab Countries}

The lack of knowledge about CRC and screening was reported as a prominent barrier for screening adherence, knowledge about CRC has proven to be an independent predictor of positive attitudes toward screening, which in-turn is related to the level of education (Tong et al., 2000). High-risk perception has been cited frequently as a predictor of CRC screening, a higher perception of risk among relatives of patients with CRC increased with the number of relatives with CRC (Santos et al., 2011).

Not all adopted strategies for colorectal cancer screening in western countriescould be undertaken in the Arab world, the culture, tradition, life style is different and could significantly influence the adherence of screening programs Literacy has been used as a variable to explain the impact of education on health behaviour in general and screening participation in particular (Paasche-Orlow and Wolf, 2007).

What is needed is education designed to modify life styles known to have a risk for cancer, coupled with policy designed to encourage healthier living, in addition to developing and sustaining comprehensive colorectal cancer early diagnosis and management with governmental health services on the basis of evidence based polices and supported by sound health care financing schemes, adequate human resources. However the engagement of national governments to finance and to develop and effective cancer health services vary widely between countries, it is country specific, each country has its own culture and stage of economic development.

All programs should be directed to high risk group i.e. first degree relatives of colo-rectal cancer case, they should be our target and not the whole community, these strategies could be achieved through: $i$ ) Primary care providers; who have an important role across cancer continuum, from encouraging screening and accurately diagnosed patients to providing care after treatment. The higher cancer screening participation rates are associated with greater involvement of primary care. In addition to the role of NGOs for encouraging and increasing participation in the colorectal cancer screening and removing obstacles and combating negative attitudes such as such as anxiety, embarrassment, disinterest, fear of cancer or screening tests, lack of time, feeling healthy, subjective perception of pain or danger, discomfort and apprehensions about bowel preparation. ii) Peer education; was found to be effective for increasing the knowledge, beliefs, and practice of women related to breast cancer (Gozum et al., 2010). Peers can reinforce learning through ongoing contact. Peer education can be used to improve early diagnosis of colorectal cancer and increase awareness in asymptomatic first degree relatives of colorectal cancer patients. iii) Education and counseling from providers or community health workers in addition to information brochures can increase awareness of colorectal cancer risk and screening options, counter negative perceptions of screening and addressing various topics related to cancer screening knowledge, barriers, risks, and outcomes. iv) Media campaigns can be used to increase cancer screening behaviour, especially when combined with efforts from other community groups.

\section{Conclusions}

The incidence of colorectal cancer in the developing Arab world in increasing, though the figures are lower than that reported from the developed countries, no activities have been adopted for national screening programs in such countries. Not only that lifestyle is critically important to the development of cancer, but that individuals and communities, can do much to prevent cancer. What is needed is health education to modify risky lifestyle, and to increase motives and enhance positive attitudes towards early screening especially amongst high risk groups, first degree relatives, in addition to policy designed to encourage healthier living. Results from genetic tests can lead to more efficient and appropriate use of screening and surveillance regimens, which will not be available in the Arab world in the near future

\section{References}

Ahmed F (2013). Barriers to colorectal cancer screening in the developing world: the view from Pakistan. World $J$ Gastrointest Pharmacol Ther, 4, 83-85.

Alamri F, Saeedi FY, Kassim KA (2014). Dietary and other risk factors for colo-rectal cancer in Saudi Arabia. Journal of Medicine and medical sciences, 5, 222-29.

American Society of Clinical Oncology (2003). policy statement update: genetic testing for cancer susceptibility. J Clin Oncol, 21, 2397-406.

Arafa MA, Sallam S and Jeirsat S (2011). Colorectal cancer screening amongst first degree relatives of colon cancer cases 
in jordan. Asian Pac J Cancer Prev, 12, 1007-11.

Arafa MA, Waly MI, Jriesat S, et al (2011). Dietary and lifestyle characteristics of colorectal cancer in jordan: a case-control study. Asian Pac J Cancer Prev, 12, 1931-36.

Barchana M (2006). Colorectal cancer - Middle East cancer consortium; 2006.

Broderick P, Carvajal-Carmona L, Pittman AM, et al (2007). A genome-wide association study shows that common alleles of SMAD7 influence colorectal cancer risk. Nature Genet, 39, 1315-7.

Carpten JD, Faber AL, Horn C, et al (2007). A transforming mutation in the pleckstrin homology domain of AKT1 in cancer. Nature, 448, 439-444.

Colon Cancer. Accessed at http://www.cags.org.ae/ gme2cancersencolon.pdf

Colorectal cancer, Facts \& Figures 2014-2016. accessed at http:// www.cancer.org/acs/groups/content/documents/document/ acspc-042280.pdf

Dolatkhah R, Hossein MS, Jabbarpour MB, et al (2015). Colorectal cancer in Iran: molecular epidemiology and screening strategies. J Cancer Epidemiol, 643020.

Dragovich T, Tsikitis V (2012). Colon Cancer, Adenocarcinoma; 2012. February [Internet].

Drummond JT, Li GM, Longley MJ, et al (1995). Isolation of an hMSH2-p160 heterodimer that restores DNA mismatch repair to tumor cells. Sci. 268, 1909-12.

Farrington SM, Tenesa A, Barnetson R, et al (2005). Germline susceptibility to colorectal cancer due to base-excision repair gene defects. Am J Hum Genet, 77, 112-9.

Fishel R, Lescoe MK, Rao MRS, et al (1993). The human mutator gene homolog MSH2 and its association with hereditary nonpolyposis colon cancer. Cell, 75, 1027-38.

Galal O (2003). Nutrition related health patterns in the Middle East. Asia Pac J Clin Nutr, 12, 337-43.

GCC Cancer Registry. ten years cancer Incidence among nationals in GCC States, 1998-2007.

Giovannucci E (2002). Modifiable risk factors for colon cancer. Gastroenterol Clin North Am, 31, 925-43.

Goel A and Boland CR (2012). Epigenetics of colorectal cancer. Gastroenterol, 143, 1442-60.

Gozum S, Karayurt O, Kav S, et al (2010). Effectiveness of peer education for breast cancer screening and health beliefs in eastern Turkey. Cancer Nurs, 33, 213-20

Guda K, Moinova H, He J, et al (2009). Inactivating germ-line and somatic mutations in polypeptide $\mathrm{N}$-acetylgalactosaminyltransferase 12 in human colon cancers. Proc Nat Acad Sci, 106, 12921-5.

Hogan NM, Hanley M, Hogan AM, et al (2015). Awareness and uptake of family screening in patients diagnosed of colorectal cancer at young age. Gastroenterol Res Pract, 194931.

Horii A, Han HJ, Sasaki S, et al (1994). Cloning, characterization and chromosomal assignment of the human genes homologous to yeast PMS1, a member of mismatch repair genes. Biochem Biophys Res. Commun, 204, 1257-64.

Ilyas M, Tomlinson IPM, Rowan A, et al (1997). Beta-catenin mutations in cell lines established from human colorectal cancers. Proc Nat Acad Sci, 94, 10330-4.

Kilpivaara O, Alhopuro P, Vahteristo P, et al (2006). CHEK2 I157T associates with familial and sporadic colorectal cancer. J Med Genet, $\mathbf{4 3}, 34$.

Kinzler KW, Nilbert MC, Vogelstein B, et al (1991). Identification of a gene located at chromosome $5 \mathrm{q} 21$ that is mutated in colorectal cancers. Science, 251, 1366-70.

Leenders M, Leufkens AM, Siersema PD, et al (2014). Plasma and dietary carotenoids and vitamins $\mathrm{A}, \mathrm{C}$ and $\mathrm{E}$ and risk of colon and rectal cancer in the european prospective investigation into cancer and nutrition. Int J Cancer, $\mathbf{1 3 5}$,
2930-9.

Lipkin SM, Wang V, Stoler DL, et al (2001). Germline and somatic mutation analyses in the DNA mismatch repair gene MLH3: evidence for somatic mutation in colorectal cancers. Hum. Mutat, 17, 389-96.

Liu W, Dong X, Mai M, et al (2000). Mutations in AXIN2 cause colorectal cancer with defective mismatch repair by activating beta-catenin/TCF signalling. Nature Genet, 26, 146-7.

Markowitz S, Wang J, Myeroff L, et al (1995). Inactivation of the type II TGF-beta receptor in colon cancer cells with microsatellite instability. Science, 268, 1336-8.

Misale, S, Yaeger R, Hobor S, et al (2012). Emergence of KRAS mutations and acquired resistance to anti-EGFR therapy in colorectal cancer. Nature, 486, 532-6.

Missaoui N, Jaidaine L, Ben Abdelkader A, et al (2011). Colorectal Cancer in Central Tunisia: Increasing Incidence Trends over a 15-Year Period. Asian Pac J Cancer Prev, 12, 1073-6.

Musaiger AO, Miladi S (2001). Food consumption patterns and dietary habits in the Arab countries of Gulf. 2001; FAO/RNE, UAE University, Al-Ain UAE.

Nicolaides NC, Kinzler KW and Vogelstein B (1995). Analysis of the 5-prime region of PMS2 reveals heterogeneous transcripts and a novel overlapping gene. Genomics, 29, 329-34.

Nishisho I, Nakamura Y, Miyoshi Y, et al (1991). Mutations of chromosome 5q21 genes in FAP and colorectal cancer patients. Science, 253, 665-9.

Paasche-Orlow MK and Wolf MS (2007). The causal pathways linking health literacy to health outcomes. Am J Health Behav, 31, 19-26.

Papadopoulos N, Nicolaides NC, Wei YF, et al (1994). Mutation of a mutL homolog in hereditary colon cancer. Science, 263, 1625-9.

Qumseya BJ, Tayem YI, Dasa OY, et al (2014). Barriers to colorectal cancer screening in Palestine: a national study in a medically underserved population. Clin Gastroenterol Hepatol, 12, 463-9.

Rajagopalan H, Bardelli A, Lengauer C, et al (2002). RAF/RAS oncogenes and mismatch-repair status. (Letter) Nature, 418, 934.

Samuels Y, Wang Z, Bardell A, et al (2004). High frequency of mutations of the PIK3CA gene in human cancers. Scienc, 304, 554.

Santos EM, Lourenco MT, Rossi BM (2011). Risk perception among Brazilian individuals with high risk for colorectal cancer and colonoscopy. Hereditary Cancer Clin Practice, 9.

Shamseddine A, Saleh A, Seoud M, et al (2014). Cancer trends in Lebanon: a review of incidence rates for the period of 20032008 and projections until 2018. Popul Health Metr, 12, 4.

Tomlinson I, Webb E, Carvajal-Carmona L, et al (2007). A genome-wide association scan of tag SNPs identifies a susceptibility variant for colorectal cancer at 8q24.21. Nature Genet, 39, 984-8.

Tong S, Hughes K, Oldenburg B, et al (2000). Sociodemographic correlates of screening intention for colorectal cancer. Australian New Zealand J Public Health, 24, 610-4.

Vatandoost N, Ghanbari J, Mojaver M, et al (2015). Early detection of colorectal cancer: from conventional methods to novel biomarkers. J Cancer Res, 2015. [Epub ahead of print].

Veruttipong D, Soliman AS, Gilbert SF, Blachley et al (2012). Age distribution, polyps, and rectal cancer in the Egyptian population-based cancer registry. World J Gastroenterol, 3997-4003.

Waly MI, Arafa MA, Jriesat SB, et al (2012). Folate and vitamin B12 deficiency is associated with colorectal cancer in Jordan. 
Mostafa A Arafa and Karim Farhat

Int J Nutrition, Pharmacol Neurodegenerative Dis, 2, 57-60.

Weyl H, Yackzan S, Ross K, et al (2015). Understanding colorectal screening behaviors and factors associated with screening is a community hospital setting. Clin J Oncol Nurs, 19, 89-93.

WHO (2002). National cancer control programmes, Policies and managerial guidelines, $2^{\text {nd }}$ edition.

World Cancer Research Fund (WCRF)/American Institute for Cancer Research (AICR) (2008). Food, nutrition and the prevention of cancer; a global perspective. Washington, DC: American Institute for Cancer Research. 2008.

World cancer research Fund International. Colorectal cancer statistics. 